\title{
Amlodipine-Induced Eczematous Drug Eruption
}

Amr Ali, MD', - Sanaa Mahmoud, MD, PhD ${ }^{3,4}$ - Jay Nguyen, BS

A 63-year-old man with hypertension was prescribed amlodipine for blood pressure control. Three days after initiating treatment, he developed an extremely pruritic rash on his back. Two steroid injections were administered at an urgent care clinic 1 day apart, and the patient was started on methylprednisolone. He experienced little improvement of his symptoms and was referred by his primary care physician for further allergy evaluation, where he presented to our clinic.

The patient had been living by himself, denied use of new soaps or detergents, denied use of lotions or creams, and denied any other recent skin lesions, atopic symptoms, or urticaria. At the time of onset of the rash, the patient had taken levothyroxine, pravastatin, omeprazole, metoprolol, and amlodipine.

\section{Physical examination}

Multiple discrete discoid, erythematous, maculopapular patches with fine scales covered most of the back. The

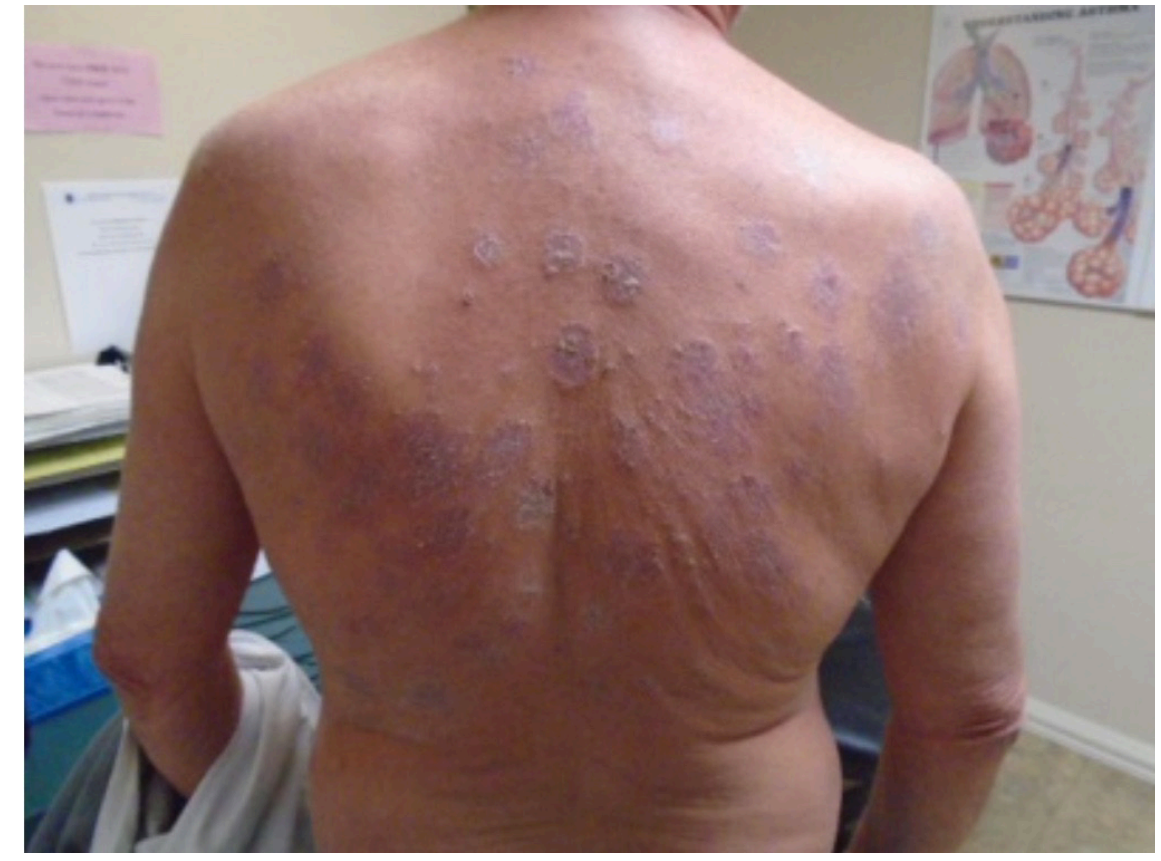

Figure 1. Multiple discrete discoid, erythematous, maculopapular patches with fine scales were noted on the patient's back. skin in between each lesion was clear (Figure 1). Neither mucous membrane

\section{AFFILIATIONS:}

'Elmhurst Hospital Center, Icahn School of Medicine at Mount Sinai, New York, New York ${ }^{2}$ Research Fellow, Department of Pediatrics, Ains Shams University, Egypt

${ }^{3}$ University of Pikeville-Kentucky College of Osteopathic Medicine, Pikeville, Kentucky

${ }^{4}$ Faculty of Medicine, Ains Shams University, Egypt

${ }^{5}$ Lincoln Memorial University-DeBusk College of Osteopathic Medicine, Harrogate, Tennessee

\section{CITATION:}

Ali A, Mahmoud S, Nguyen J. Amlodipine-induced eczematous drug eruption. Consultant. Published online February 2, 2022. doi:10.25270/con.2022.02.00002

Received August 5, 2021. Accepted August 30, 2021.

\section{DISCLOSURES:}

The authors report no relevant financial relationships.

\section{CORRESPONDENCE:}

Sanaa Mahmoud, MD, PhD, University of Pikeville-Kentucky College of Osteopathic Medicine, 147 Sycamore Street, Pikeville, Kentucky 41501 (smahmoudcairo@gmail.com involvement nor systemic symptoms were reported.

The amlodipine was discontinued and replaced with hydrochlorothiazide. $\mathrm{He}$ was prescribed $\mathrm{H} 1$ - and $\mathrm{H} 2$-antagonists, as well as a tapering course of prednisone. A skin biopsy was performed and sent for evaluation. An aeroallergen skin test and food allergy skin test were performed, results of which did not show any significant sensitization. Patch testing was also performed, but results did not show any significant positive reactions.

After stopping a second course of prednisone, the patient again experienced pruritus but with very gradual improvement of the rash. At that point, the rash was further controlled by topical steroids mixed with over-the-counter topical emollients. 


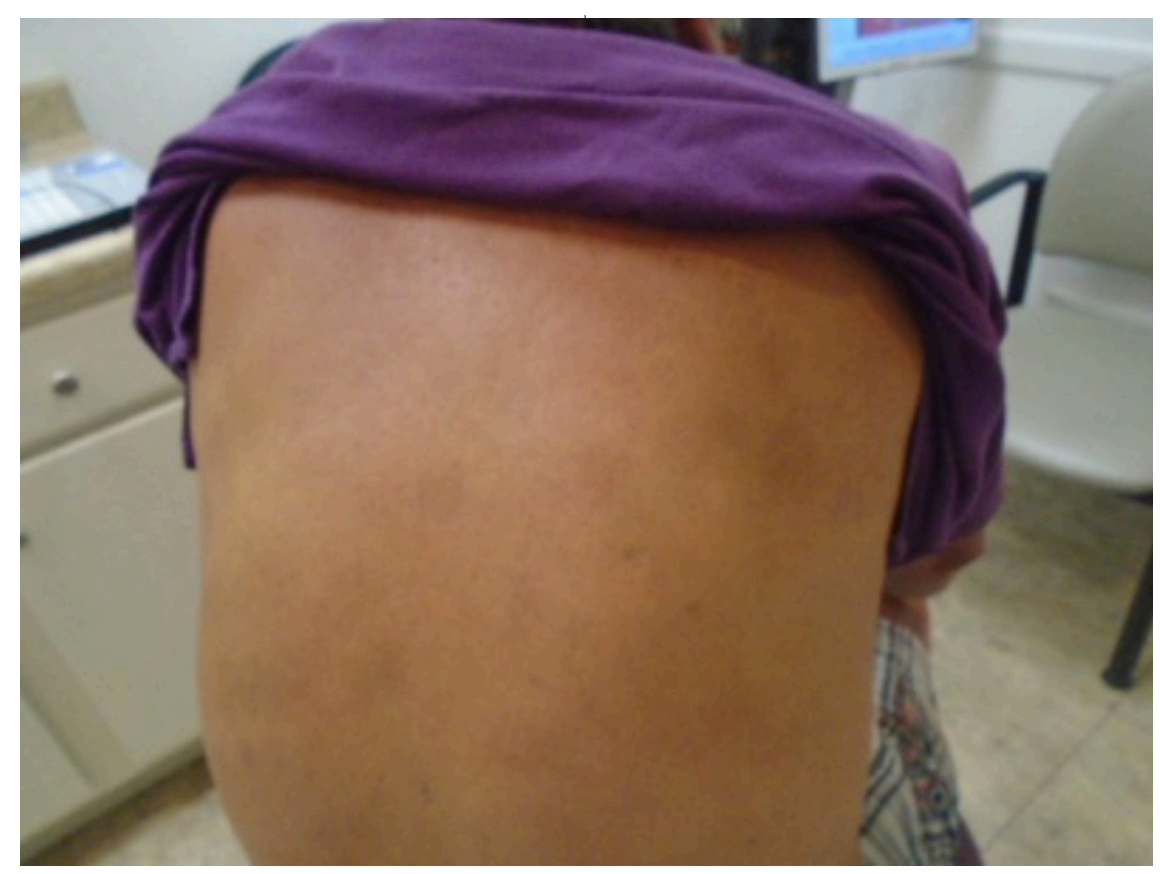

Figure 2. The patient's back after 8 months of withdrawing amlodipine.

The results of the skin biopsy returned and revealed the presence of hyperkeratosis with parakeratosis, serum overlying the epidermis, spongiosis, irregular psoriasiform hyperplasia, and a brisk superficial perivascular lymphocytic infiltrate with eosinophils. These results were suggestive of subacute eczematous and spongiotic dermatitis of a drug reaction with no evidence of vasculitis. Upon withdrawal of amlodipine, the patient's rash gradually improved to complete resolution without any residue over 8 months (Figure 2).

\section{Discussion}

Calcium channel blockers (CCBs) are frequently used to treat cardiovascular diseases such as hypertension; amlodipine is one of the most frequently prescribed CCBs. ${ }^{1}$ Previous studies of adverse drug reactions associated with antihypertensive medications have shown that CCBs were the most common cause of adverse drug reactions. ${ }^{2}$ Both allergic and nonallergic adverse drug reactions have been reported, such as flushing, gingival hyperplasia, gynecomastia, and cutaneous adverse drug reactions. ${ }^{3}$
There is a wide spectrum of cutaneous reactions to $\mathrm{CCBs}$, ranging from isolated maculopapular rash to erythema multiforme major and toxic epidermal necrolysis. ${ }^{4}$ Among the CCBs, diltiazem has the highest rate of triggering cutaneous hypersensitivity, while dihydropyridines such as amlodipine have lower rates. ${ }^{5}$ Drug-induced hypersensitivity reactions to amlodipine are rare, but associations between CCBs and cutaneous adverse drug reactions have been reported..$^{6-8} \mathrm{We}$ describe a case of a discoid eczematous adverse drug reaction that started a few days after initiation of amlodipine for hypertension and took 8 months after discontinuation of the drug to resolve completely.

Amlodipine was suggested as the triggering agent because of the temporal relation between its initiation and the development of our patient's rash. Causality was supported by a biopsy, which showed findings consistent with a possible drug reaction. Contact dermatitis was considered but excluded by negative skin patch testing. Psoriasis was considered based on histopathologic findings but was excluded because of a lack of typical physical examination findings, such as silvery scale.

Cutaneous adverse drug reactions triggered by amlodipine have been rarely reported. Flushing, a nonallergic process, is the most common cutaneous adverse effect; urticaria and an erythematous maculopapular rash are the most common allergic reactions. ${ }^{9}$

Although CCB-induced cutaneous adverse drug reactions have been studied, most studies are limited to a few case reports. One study was conducted between January 2004 and December 2010 in Thailand and showed that out of 996,583 CCB prescriptions, only 46 patients had developed cutaneous adverse drug reactions, with maculopapular rash being the most common dermatologic manifestation at $41.7 \% .^{3}$ Diltiazem had the highest rate of cutaneous adverse drug reactions per million prescriptions compared with other CCBs. ${ }^{3}$

It is of clinical importance to mention the duration of complete resolution of our patient's rash. Eight months is considerably long compared with the average duration of resolution of most drug-induced eruptions (ie, 1-2 weeks). A search of the literature did not return any cases of prolonged drug eruption beyond a couple months.

\section{Conclusions}

Amlodipine-induced eczematous cutaneous adverse drug reactions should not be considered uncommon and should be considered when multiple drugs are being taken. Moreover, patients should be advised of the possible prolonged duration of resolution of the rash regardless of additional medical management of the symptoms.

\section{References}

1. Elliott WJ, Ram CVS. Calcium channel blockers. J Clin Hypertens (Greenwich). 2011;13(9):687-689. https://doi.org/10.1111/ j.1751-7176.2011.00513.x

2. Ross SD, Akhras KS, Zhang S, Rozinsky M, Nalysnyk L. Discontinuation of antihypertensive drugs due to adverse events: a 


\section{PHOTOCLINIC}

systematic review and meta-analysis. Pharmacotherapy. 2001;21(8):940-953. https:// doi.org/10.1592/phco.21.11.940.34520

3. Tuchinda P, Kulthanan K, Khankham S, Jongjarearnprasert K, Dhana N. Cutaneous adverse reactions to calcium channel blockers. Asian Pac J Allergy Immunol. 2014;32(3):246-250. https://doi. org/10.12932/ap0380.32.3.2014

4. Ioulios $\mathrm{P}, \mathrm{Charalampos} \mathrm{M}$, Efrossini T. The spectrum of cutaneous reactions associated with calcium antagonists: a review of the literature and the possible etiopathogenic mechanisms. Dermatol Online J. 2003;9(5):6. https://doi.org/10.5070/D39h$3065 \mathrm{pb}$

5. Knowles S, Gupta AK, Shear NH. The spectrum of cutaneous reactions associated with diltiazem: three cases and a review of the literature. J Am Acad Dermatol. 1998;38(2 Pt 1):201-206. https://doi. org/10.1016/s0190-9622(98)70241-5

6. Kuruvilla ME, Sanan N. Amlodipine-induced angioedema: An unusual complication of a common medication. Allergy Rhinol (Providence). 2018;9:2152656718764139. https:// doi.org/10.1177/2152656718764139

7. Yoo J, Jue MS. Intractable pruritus with chronic eczema in an elderly patient caused by long-term intake of calcium channel blocker. Contact Dermatitis. 2017;77(5):339340. https://doi.org/10.1111/cod.12832

8. Joly P, Benoit-Corven C, Baricault S, et al. Chronic eczematous eruptions of the elderly are associated with chronic exposure to calcium channel blockers: results from a case-control study. J Invest Dermatol. 2007;127(12):2766-2771. https:// doi.org/10.1038/sj.jid.5701018

9. Rezaković S, Počanić L. Cutaneous adverse drug reactions induced by amlodipine. Cardiologia Croatica. 2014;9(7-8):314. https:// doi.org/10.15836/ccar.2014.314 